\title{
Whole-tumor apparent diffusion coefficient measurements in nephroblastoma: Can it identify blastemal predominance?
}

Abstract

Purpose

To explore the potential relation between whole-tumor apparent diffusion coefficient (ADC) parameters in viable parts of tumor and histopathological findings in nephroblastoma.

Materials and Methods

Children $(n=52)$ with histopathologically proven nephroblastoma underwent diffusionweighted magnetic resonance imaging (MRI) (1.5T) before preoperative chemotherapy. Of these, 25 underwent an additional MRI after preoperative chemotherapy, shortly before resection. An experienced reader performed the whole-tumor ADC measurements of all lesions, excluding nonenhancing areas. An experienced pathologist reviewed the postoperative specimens according to standard SIOP guidelines. Potential associations between ADC parameters and proportions of histological subtypes were assessed with Pearson's or Spearman's rank correlation coefficient depending on whether the parameters tested were normally distributed. In case the Mann-Whitney U-test revealed significantly different $A D C$ values in a subtype tumor, this $A D C$ parameter was used to derive a receiver operating characteristic (ROC) curve.

Results

The 25th percentile ADC at presentation was the best $A D C$ metric correlated with proportion of blastema (Pearson's $r=-0.303, P=0.026$ ). ADC after preoperative treatment showed moderate correlation with proportion stromal subtype at histopathology $(r=0.579$, $P=0.002)$. By ROC analysis, the optimal threshold of median ADC for detecting stromal subtype was $1.362 \times 10-3 \mathrm{~mm} 2 / \mathrm{s}$ with sensitivity and specificity of $100 \%$ (95\% confidence interval $[\mathrm{Cl}] 0.65-1.00)$ and $78.9 \%(95 \% \mathrm{Cl} 0.57-0.92)$, respectively.

Conclusion

ADC markers in nephroblastoma are related to stromal subtype histopathology; however, identification of blastemal predominant tumors using whole-tumor ADC measurements is probably not feasible.

\section{Level of Evidence 3}

Wilms' tumor (nephroblastoma) is the most common, malignant renal tumor in children.[14] It develops from embryonic kidney cells, containing varying amounts of tissue recapitulating stages of renal development, including blastemal, epithelial, and stromal components.[1] According to the current International Society of Pediatric Oncology guidelines, the treatment in patients over the age of 6 months includes 4-6 weeks' preoperative chemotherapy, followed by surgery.[2] Overall, the outcome of patients with nephroblastoma is excellent[4] and the current focus is on identifying biological and imaging markers to further improve outcomes and reduce therapy-induced long-term sequelae in these very young patients.[4]

Apparent diffusion coefficient (ADC) may be an interesting imaging biomarker for identifying high-risk histopathology type nephroblastomas and for assessing treatment response. However, the literature on the role of diffusion-weighted imaging (DWI) in nephroblastoma is limited. A few studies have identified associations between ADC values and histopathological findings in a variety of pediatric extracranial mass lesions.[5-11] Humphries et al found a significant relationship between cellularity and ADC value in a range of pediatric tumors.[5] Some studies have reported considerable differences in ADC value between benign and malignant abdominal mass lesions.[6, 7] McDonald et al 
demonstrated that changes in ADC in pediatric abdominal masses during therapy are measurable.[8] A recent preclinical study has reported that with mathematical modeling it is possible to identify subtypes of ADC in nephroblastoma.[9] However, the implication of the $A D C$ measurements in routine clinical practice has not yet been determined. Therefore, we searched for ADC measurements that might provide clinically relevant information in patients with nephroblastoma. More specifically, we investigated if whole-tumor ADC measurements could help identify high-risk lesions that require intensification of treatment, for instance, blastemal predominant tumors.

The aim of this retrospective explorative study was to investigate specific patterns in ADC change during preoperative treatment and to study potential ADC metrics that are related to the different histopathological subtypes in nephroblastoma using a previously published reproducible method.

\section{Materials and Methods}

Patients

The Research Ethics Committee of our institution waived the need for formal ethics approval, or informed consent for this retrospective study, and in particular since all examinations included in the study were clinically indicated and had been conducted in accordance with the normal standard of care in our institution. Great Ormond Street Hospital for children is a tertiary referral center for nephroblastoma and a quartiary referral center for bilateral nephroblastoma. Between July 2007 and February 2014, 72 consecutive children were considered for inclusion. Inclusion criteria were histologically proven nephroblastoma, treatment according to the SIOP-RT 2001 protocol (preoperative treatment followed by surgical resection), complete MRI study including DWI at staging, and availability of the tumor specimen for histopathological review. Exclusion criteria were severe artifacts at DWI or postcontrast images, tumor size less than $6 \mathrm{~cm} 3$ (which would render ADC estimates less reliable), or predominantly cystic lesions (since ADC of cyst content is clinically uninteresting). In addition to DWI, a complete MRI study included at least $T 1$-weighted sequences pre- and postcontrast medium administration. This was in order to enable exclusion of nonenhancing parts (likely necrotic parts) of tumor from the ADC analysis.

Twenty patients were excluded due to the following: incomplete MRI study $(n=8)$; diagnostically relevant artifacts $(n=3)$; no histopathological specimen available for review $(n=3)$; lesion too small $(n=3)$; predominantly cystic $(n=2)$; non-Wilms' renal tumor $(n=1)$. The final study population consisted of the remaining 52 patients (mean age 2.6 years, range 1 months to 12.1 years, 24 males, Table 1). The preoperative treatment consisted of 4 weeks of chemotherapy with vincristine and actinomycin $D$ for localized tumors. Children with metastases at diagnosis received 6 weeks of preoperative therapy with three drugs, including doxorubicin, according to the guidelines of the SIOP-RT 2001 protocol.

Table 1. Characteristics of Included Patients In 25 patients, complete diagnostic MRI including DWI was performed after the preoperative therapy, shortly before surgical resection for restaging purposes. Some of the scans had previously been included in a mathematical modeling study.[9] We used a clinically driven approach to investigate if whole-tumor ADC measurements could help in identifying blastemal predominant tumors.

The flow diagram according to standards for reporting diagnostic accuracy studies (STARD) is illustrated in Fig.

MRI Acquisition 
Abdominal MRI in our institution during the study period was performed with a 1.5T MRI system (Magnetom Avanto; Siemens, Erlangen, Germany). The imaging protocol consisted of axial and coronal T2-weighted short-tau inversion recovery, fat-suppressed axial pre- and postcontrast $T 1$-weighted turbo spin-echo, and axial DWI. DWI was acquired with at least the following b-values: $0,50,250,500$, and $1000 \mathrm{~s} / \mathrm{mm} 2$. The imaging parameters are displayed in Table 2. ADC maps were automatically generated by the scanner operating system.

Table 2. Scan Parameters at 1.5T MRI for Suspected Renal Tumor and for Imaging of Nephroblastoma After Neoadjuvant Chemotherapy

Children were awake, sedated, or under general anesthesia, depending on their ability to cooperate. All children were screened for contraindications for MRI, for use of intravenous contrast agents, and for intravenous hyoscine butylbromide. No oral contrast agents were used. Gadoterate meglumine (Dotarem; Guerbet Laboratories, Roissy, France) was administered at an intravenous dose of $0.05 \mathrm{mmol} / \mathrm{kg}$ body weight; $0.4 \mathrm{mg} / \mathrm{kg}$ body weight of hyoscine butylbromide (Buscopan, Boehringer Ingelheim, Bracknell, UK) was given intravenously to reduce peristaltic artifacts.

Image Analysis

The anonymized MR datasets were transferred to DICOM software Osirix v. 5.5.2 (Pixmeo SARL, Bernex, Switzerland). A pediatric radiologist (A.S.L. with 9 years of experience in body MRI), who was blinded to clinical patient data and histopathology reports, performed the ADC measurements. The pretreatment images were at the readers' disposal when analyzing the posttreatment images, in order to compare and identify the location of tumor. The reliability of this method had already been demonstrated to be sufficient,[12] and therefore it was deemed unnecessary to have more than one reader.

Whole-Tumor ADC Measurements

A freehand region of interest (ROI) was carefully drawn on the ADC map using the postcontrast and T2-weighted images to guide the outline of the entire tumor at each consecutive tumor-containing slice, excluding peritumoral edema. To reduce the partial voluming effect, we included the slices the where tumor area was $>50 \%$ of the adjacent more central slice and where the tumor area was at least $3 \mathrm{~cm} 3$.

Areas of tumor with no or very low enhancement were excluded from further analysis, because these are thought to represent necrosis, hemorrhage, or cystic elements. These components can skew the ADC location parameters if included.[12] This method was described in detail previously.[12]

Whole-tumor ADC values before subtraction was documented in order to study the effect of subtraction. The DICOM software automatically calculated the tumor volume estimates from the whole-tumor ROIs. Viable portions of tumor were calculated by dividing the number of pixels after subtraction of less-enhancing parts of the lesions by the number of pixels before subtraction.

Recent studies have shown that volume of blastema has prognostic value with respect to event-free and overall survival.[3] Therefore, we analyzed the volume of blastema in preoperative treated lesions and ADC parameters. The estimated volume of blastema (reference standard) was calculated by the following formula: volume provided by wholetumor ROI before subtraction $\times$ (1- proportion of chemotherapy induced changes at histopathology) $\times$ the proportion of blastema at histopathology.

Histopathological Review

One consultant pediatric histopathologist (N.J.S. with more than 15 years' experience with 
pediatric tumor histopathology) reviewed histological sections from the surgically resected tumors of all included patients. The percentage of chemotherapy-induced changes, percentage of stromal, epithelial, and blastemal elements, overall tumor type, and stage were recorded for every tumor, blinded to MRI findings. The SIOP-WT 2001 criteria for subtyping nephroblastoma were used (Table 3).[1]

Table 3. Histological Criteria for Nephroblastoma Tumor Subtyping in SIOP WT 2001 Statistical Analysis

The Shapiro-Wilk test was used to check whether the variables tested could reasonable be assumed normally distributed. W more than 0.90 was considered to represent normal distribution.

The Wilcoxon Rank test was used to test if the ADC median pre vs. ADC median post and $A D C$ median with subtraction vs. without subtraction were significantly different.

Potential associations between ADC parameters and proportions of histological subtypes were assessed with Pearson's or Spearman's rank correlation coefficient, depending on whether the parameters tested were normally distributed.

The ADC values of tumors with $>2 / 3$ stromal histopathology were compared with the ADC values of the other subtypes using the Mann-Whitney $U$-test. In case the test revealed a significant difference, diagnostic performance was analyzed with construction a receiver operator characteristic (ROC) curve with calculation of the area under the curve (AUC) along with $95 \%$ confidence intervals (Cls). The ROC curve was used to determine the optimal AUC cutoff value with corresponding sensitivity and specificity. $P<0.05$ was considered significant. SPSS v. 22 for Mac (Chicago, IL) was used for all statistical analysis.

Results

ADC With and Without Subtraction of Areas With No or Very Low Enhancement To assess if subtraction of areas with no or very little enhancement is needed in every lesion, we compared the ADC values before and after subtraction.

The differences in whole-tumor median ADC at presentation before and after subtraction of less-enhancing parts ranged between -0.28 and $0.28 \times 10-3 \mathrm{~mm} 2 / \mathrm{s}$, with a median difference of $0.009 \times 10-3 \mathrm{~mm} 2 / \mathrm{s}$ (54 lesions in 52 patients). In five patients the difference was more than $0.10 \times 10-3 \mathrm{~mm} 2 / \mathrm{s}$. In 24 patients there was no difference.

The differences before and after subtraction for median ADC after preoperative treatment were more distinct ( 25 lesions in 25 patients). The median difference was $0.09 \times 10-3$ $\mathrm{mm} 2 / \mathrm{s}$ (range -0.50 to $0.62 \times 10-3 \mathrm{~mm} 2 / \mathrm{s}$ ). In eight patients there was no difference. In seven patients the difference was more than $0.10 \times 10-3 \mathrm{~mm} 2 / \mathrm{s}$.

Whole-Tumor MRI Parameters and Histopathological Response

Change in size is often used to assess treatment response in oncology. Therefore, we studied the relation between volume change during preoperative treatment and chemotherapy-induced changes seen at histopathology. The median volume of pretreated lesions was $424.9 \mathrm{~cm} 3$ (range 10.7-1157.8, interquartile range [IQR] 447.7). After treatment the median volume of lesions was $204.5 \mathrm{~cm} 3$ (6.21-1208.0; IQR 209.9). The difference in volume (median 164.4 (-464.6-1023.8; IQR 190.3)) was not related to chemotherapyinduced changes at histopathology (Spearman's' $r=0.099 ; 95 \% \mathrm{Cl}-0.173-0.357, P=0.637$ ). The proportion of enhancing (viable) parts of lesions decreased after treatment (median viable portion at presentation 0.95 (range 0.65-1.00); IQR 0.14 and median viable portion after preoperative treatment 0.86 (range $0.25-1.00$; IQR 0.31)).

The enhancing portion after treatment showed a strong inverse relation with chemotherapy-induced changes at histopathology $(r=-0.775 ; 95 \% \mathrm{Cl}-0.895$ to $-0.548, P<$ 
$0.0005)$

Median ADC Before and After Preoperative Treatment

To assess if we were able to measure treatment response other than shrinkage and necrosis, we analyzed the ADC parameters at presentation and after preoperative treatment after subtraction of the less-enhancing parts of lesions. The mean ADC of the 54 whole-tumor median ADC at presentation was $0.945 \times 10-3 \mathrm{~mm} 2 / \mathrm{s}$ (SD 0.232; range 0.641$1.416 \times 10-3 \mathrm{~mm} 2 / \mathrm{s})$. In 25 of 54 lesions that were available for analysis after preoperative chemotherapy, the mean ADC was $1.342 \times 10-3 \mathrm{~mm} 2 / \mathrm{s}$ (SD 0.350; range 0.781-1.951 $\times$ 10-3 mm2/s). ADC at presentation was significantly lower compared to ADC after preoperative treatment $(Z-4.276, P<0.0005)$ (Fig. 2).

ADC Parameters and Histopathology Findings

The distribution of median ADC after treatment per histopathological subtype is illustrated in Fig. 3, which also shows the distribution of the changes in median ADC after preoperative treatment by histopathology type. Figure 4 illustrates examples of histopathological subtypes with MRI appearances before and after preoperative treatment.

However, only a few blastemal and epithelial predominant tumors were represented in this cohort. Therefore, we also studied the relation between the proportion of each histopathological component within the lesion and ADC parameters.

\section{Blastemal Histopathology}

With the small number of patients we included, there was no significant correlation between median ADC at presentation and the proportion of blastema $(r=-0.248 ; 95 \% \mathrm{Cl}-$ $0.483-0.021, P=0.070$ ) (Table 4). However, the 25th percentile ADC and proportion of blastema in 54 lesions at staging showed a weak but significant correlation ( $r=-0.303 ; 95 \%$ $\mathrm{Cl}-0.527$ to $-0.039, P=0.026$ ) (Table 4). Figure 5 shows the relationship of 25 th percentile $A D C$ at presentation and the proportion of blastema and epithelial subtype at histopathology. This plot illustrates the overlap in low ADC values between lesions with relative high blastemal and epithelial proportions. The correlation between the 25th percentile ADC and blastemal proportion in 25 lesions after treatment was not significant $(r=-0.366 ; 95 \% \mathrm{Cl}-0.664$ to $-0.034, P=0.072)$.

\section{Figure 4.}

A $6 \frac{1}{2}$-year-old girl presented with a large right-sided renal tumor, histopathologically proven nephroblastoma, blastemal predominant subtype. Subtracted postcontrast axial T1-W MR image (a) and corresponding axial ADC map (c) at presentation show a large, homogenous tumor with relatively low ADC value (median ADC $0.806 \times 10-3 \mathrm{~mm} 2 / \mathrm{s}$; volume $707.1 \mathrm{~cm} 3$ ). After preoperative treatment $(\mathbf{b}, \mathbf{d})$ there is shrinkage of the lesion and an increase in median ADC value (volume $115.7 \mathrm{~cm} 3$; median ADC before subtraction: $0.935 \times 10-3$ $\mathrm{mm} 2 / \mathrm{s}$, median ADC after subtraction $1.052 \times 10-3 \mathrm{~mm} 2 / \mathrm{s} ; 25$ th\% ADC after subtraction $0.669 \times 10-3 \mathrm{~mm} 2 / \mathrm{s})$. The areas of tumor with the very low ADC represent nonviable parts, as these areas show no contrast-enhancement on subtracted postcontrast axial T1-W MR image. MR images in a 1-month-old boy with right-sided renal nephroblastoma, epithelial subtype show the relatively low ADC (subtracted postcontrast axial T1-W image (e), ADC map (g); median ADC $0.664 \times 10-3 \mathrm{~mm} 2 / \mathrm{s})$ at presentation. After treatment there is some shrinkage of the lesion, but with a limited shift in median ADC (subtracted postcontrast axial T1-W image (f), ADC map (h); median ADC $0.781 \times 10-3 \mathrm{~mm} 2 / \mathrm{s})$. MR images in a 1-year-old girl with left-sided renal nephroblastoma, stromal subtype illustrates the relatively high ADC value at presentation (subtracted postcontrast axial T1-W image (i), ADC map (k); median 
ADC $1.401 \times 10-3 \mathrm{~mm} 2 / \mathrm{s})$. There is an increase in size of the lesion after preoperative treatment with an increase in ADC value (subtracted postcontrast axial

Scatterplot shows the relationship between 25th percentile ADC $(\times 10-3 \mathrm{~mm} 2 / \mathrm{s})$ before preoperative treatment and percentage of blastemal (black dots) and epithelial (white dots) proportion of subtype at histopathology. This plot illustrates the overlap in low ADC values between lesions with relative high blastemal and epithelial proportions.

With the limited included patients, there was no significant correlation between volume of blastema and 25th percentile ADC at presentation $(r=-0.378 ; 95 \% \mathrm{Cl}-0.586$ to -0.123 , $P=0.062$ ).

\section{Stromal Histopathology}

The median ADC showed a moderate correlation with proportion of stromal histology (at presentation: $r=0.401 ; 95 \% \mathrm{Cl} 0.15-0.603, P=0.003$ and after treatment: $r=0.579 ; 95 \% \mathrm{Cl}$ $0.239-0.792, P=0.002$ ) (Table 4).

The median ADC after preoperative treatment in lesions with $>2 / 3$ stromal histopathology $(n=7)$ was significantly higher compared to the median ADC in the other tumors $(P=0.002)$. The ROC curve is shown in Fig. 6. The AUC was 0.895 (95\% $\mathrm{Cl} 0.773-1.00)$. Using an optimal cutoff value of $1.362 \times 10-3 \mathrm{~mm} 2 / \mathrm{s}$, the sensitivity and specificity were $100 \%$ (95\% Cl $0.65-$ $1.00)$ and $78.9 \%(95 \% \mathrm{Cl} 0.57-0.92)$, respectively.

\section{Epithelial Histopathology}

The relatively limited shift in ADC after treatment appeared the best metric that correlated with proportion epithelial histopathology $(r=-0.358 ; 95 \% \mathrm{Cl}-0.659-0.043, P=0.073)$ (Table 4).

\section{Discussion}

The results of our explorative, single-center study demonstrate that 25 th percentile ADC is the most promising ADC metric related to identification of the proportion of blastema identified on histological evaluation. This finding is in concordance with previously reported results.[9] However, this weak correlation does not prove diagnostic usefulness. The existence of a linear relation disregards dispersion of observations, and any overall effect in a group of tumors cannot directly be extrapolated to an individual patient. If there is a significant overlap, for example, low ADC values that are seen in blastemal and in epithelial subtypes, the finding of low ADC may be of more limited clinical value even if there is a significant difference in the means. Therefore, the weak relationship demonstrated does not provide enough evidence that whole-tumor ADC measurements in routine clinical practice can act as a useful diagnostic tool to identify blastemal predominance. However, lesions with predominant stromal histology demonstrated a significant relation with the median ADC after preoperative chemotherapy. Furthermore, the ROC analysis showed that if an optimal cutoff value of $1.362 \times 10-3 \mathrm{~mm} 2 / \mathrm{s}$ is used, this parameter has high sensitivity (100\%) and is relatively specific (78.9\%). Stromal predominant tumors may be associated with a WT1 gene mutation frequently seen in bilateral disease.[13] The potential identification of stromal predominant tumors could therefore help in guiding preoperative therapy.

Furthermore, our results support the need for subtraction of nonviable parts of tumors when measuring whole-tumor ADC values, especially relevant for posttreatment lesions, which are more heterogeneous and has a higher likelihood of containing nonviable elements. We showed that in 7 of 25 lesions the ADC difference before and after subtraction of cystic, hemorrhagic, or necrotic parts exceeded previously reported observer variability.[12] Therefore, such differences must be regarded as diagnostically relevant. The 
importance of subtracting nonviable parts of tumor is also highlighted in a recent report in pancreatic cancer, which used a selected area ROI method that visually excluded nonviable parts of lesions. This ADC metric was reported as the strongest marker for predicting improved survival during chemotherapy treatment. However, the observer variability of their method was not reported.

Exclusion of the less-enhancing parts of the tumor allows measurement of preoperative therapy response of presumed cellular tumor elements. Our data further support previous reports[] that volume change in nephroblastoma is poorly related to response to chemotherapy, because shrinkage of tumor can be associated with high-risk histology types and conversely tumors may grow with differentiation.

Our work builds on the valuable contributions of McDonald et al and Hales et al. Hales et al used a multi-Gaussian model for ADC analysis of different nephroblastoma subtypes. They were able to identify predominant histopathological cell types in nephroblastomas. We tried to translate this preclinical work to a clinical context. Our study used a clinically driven approach studying the best location parameter, measure treatment effects beyond necrosis, and relate ADC measurements to histopathological findings.

This study has several limitations. First, it is a retrospective analysis of clinical datasets, with subsequent population bias. Second, related to the low incidence of this tumor, the number of included patients, especially in subgroups, was limited. Therefore, potential significant correlations between histopathological subtypes and ADC parameters could be obscured. Third, although we demonstrated a technically feasible method for measuring whole-tumor ADC after subtraction of less-enhancing parts, implementation in daily clinical practice could be hampered because outlining the whole-tumor volume is rather time-consuming. An alternative approach might be to place an ROI in a single section over the area of enhancing tumor with the most restricted diffusion; however, this method does probably not account for the full extent of ADC heterogeneity and is known to be less reliable. Fourth, although biopsy at presentation is performed as standard of care in our institution, whole-tumor histopathology was not available at presentation. Therefore, the comparison of ADC at presentation with the posttreatment histopathology is limited, as preoperative chemotherapy changes tumor histopathological features and distribution of subtypes. Fifth, we studied patients with localized and metastatic tumor as one cohort, although these groups receive different chemotherapeutic regimes. Finally, histopathological estimation of tumor composition is based on sampling rather than examination of the entire tumor and is therefore not perfectly objective. Further studies to assess direct correlation between ADC parameters and histopathological findings could further elucidate the potential role of diffusion-weighted imaging in nephroblastoma.

In conclusion, whole-tumor ADC markers in nephroblastoma are correlated with stromal subtype histopathology; however, reliable differentiation between epithelial and blastemal predominant types using whole-tumor ADC measurements is probably not feasible, because both subtypes show marked diffusion restriction. 